\title{
INFLUÊNCIA DO ESTADO NUTRICIONAL SOBRE O DESEMPENHO PRODUTIVO E PERFIL HORMONAL DE CORDEIRAS CONFINADAS DURANTE A FASE DE CRESCIMENTO
}

Ariane Dantas ${ }^{1}$, Eunice Oba ${ }^{1}$, Rogério Antonio Oliveira ${ }^{1}$, Felipe Rydygier de Ruediger, André Michel de Castilho, Sony Dimas Bicudo, Luana de Cássia Bicudo, Maria Fernanda Correia Silva Carrega ${ }^{2}$

Universidade Estadual Paulista Júlio de Mesquita Filho - UNESP ${ }^{1}$, Faculdade de Medicina Veterinária e Zootecnia FMVZ/UNESP, Botucatu, SP ${ }^{2}$

\section{RESUMO}

O manejo nutricional interfere na produção hormonal refletindo na eficiência produtiva e reprodutiva dos animais. Assim, o objetivo foi avaliar o efeito da diferenciação alimentar na concentração plasmática hormonal de cordeiras durante a fase de crescimento. Utilizou-se 20 cordeiras da raça Bergamácia divididas em dois grupos $(n=10)$, mantidas confinadas recebendo dieta para obtenção de ganho de peso médio diário de $150 \mathrm{~g}$ (tratamento Baixo) e 250g (tratamento Alto). A cada 14 dias realizou-se pesagem (kg), avaliação do escore de condição corporal (escala de 1 a 5) e colheitas de sangue a cada 28 dias para a determinação das concentrações plasmáticas da insulina $(\mu \mathrm{lU} / \mathrm{mL})$ e progesterona $(\mathrm{ng} / \mathrm{mL})$. As cordeiras do tratamento Alto apresentaram maior escore de condição corporal e concentrações plasmáticas de insulina, nas duas fases experimentais, contudo, os níveis plasmáticos de progesterona, não difeririam entre os grupos. Portanto, o manejo nutricional evidenciou o status nutricional e aumentou as concentrações plasmáticas de insulina de cordeiras confinadas em crescimento.

Palavras-chave: alimentação; confinamento; desempenho; perfil endócrino.

\section{INFLUENCE OF NUTRITIONAL STATUS ON PRODUCTION PERFORMANCE AND PROFILE OF HORMONE OF CONFINED LAMBS DURING THE GROWTH PHASE}

\begin{abstract}
Nutritional management interferes with hormone production reflecting the productive and reproductive efficiency of animals. The objective was to evaluate the effect of food differentiation in hormone plasma concentration of lambs during the growth phase. It was used 20 Bergamasca lambs divided into two groups $(n=10)$ and kept confined receiving diet to obtain average daily weight gain of $150 \mathrm{~g}$ (treatment Low) and 250g (treatment High). Every 14 days, weighing was performed (kg), body condition score (from 1 to 5) was aseesed and blood sampling was conducted every 28 days for determination of plasma insulin concentrations $(\mu \mathrm{lU} / \mathrm{mL}$ ) and progesterone $(\mathrm{ng} / \mathrm{mL})$. Lambs of treatment High showed higher body condition score and plasma insulin concentrations in both experimental phases, however, plasma levels of progesterone did not differ between groups. Therefore, the dietary use with larger amount of concentrated improves nutritional status and increases the plasma concentration of insulin contained lambs growth.

Keywords: feeding; confinement; performance; endocrine profile.
\end{abstract}

\section{INTRODUÇÃO}

Dentre os vários fatores que afetam o desempenho de ovinos, o manejo nutricional é considerado um de seus principais pontos, em especial os níveis de energia da dieta (NATIONAL RESEARCH COUNCIL, 2007). Segundo Eversole et al. (2000) tanto a deficiência quanto a superalimentação energética têm efeito negativo sobre o desenvolvimento corporal e atividade reprodutiva, sendo que ambas as situações significam perdas econômicas ao produtor.

A nutrição tem significativo impacto em numerosas funções metabólicas, incluindo a produção de diversos hormônios, como a insulina. De acordo com Roche et al. (2008), a insulina é o hormônio que promove o transporte e armazenamento dos combustíveis metabólicos para dentro das células, sendo um importante 
hormônio para o desenvolvimento corporal e formação de reservas no tecido periférico, podendo ser utilizado na tentativa de melhor explicar a relação entre nutrição e reprodução em ovinos.

Estímulos nutricionais podem afetar a reprodução através do seu efeito sobre o eixo hipotálamo-hipófise-gônada, interferindo assim na síntese e secreção do hormônio liberador de gonadotrofinas e dos hormônios luteinizante e folículo estimulante, bem como na produção e liberação de progesterona e estradiol (PIRES; RIBEIRO, 2011). Nesse sentido, conhecer o comportamento endócrino de cordeiras submetidas ao confinamento durante a fase de crescimento é fundamental.

Portanto, a influência do plano alimentar nos mecanismos fisiológicos é muito importante, sendo necessárias maiores investigações, visto que está associada ao sucesso da eficiência da função produtiva e reprodutiva dos animais. Desse modo, o objetivo do presente estudo foi avaliar o efeito da nutrição nas concentrações plasmáticas de insulina e progesterona de cordeiras confinadas ao longo da fase de crescimento e sua aplicabilidade prática no monitoramento do desenvolvimento corporal e puberdade.

\section{MATERIAL E MÉTODOS}

O experimento foi realizado na
Universidade Estadual Paulista (UNESP), Faculdade de Medicina Veterinária e Zootecnia, Campus de Botucatu/SP, nas instalações da Unidade de Pesquisa em Produção de Leite Ovino e teve aprovação da Comissão de Ética no uso de animais (CEUA) da Faculdade de Medicina Veterinária e Zootecnia, UNESP, Botucatu-SP (159/2010).
Utilizou-se 20 cordeiras da raça Bergamácia com idade inicial de 90 dias de idade e oriundas de partos simples. Os animais foram mantidos confinados durante toda fase experimental, em baias cobertas $(3,50 \mathrm{~m} \times$ $1,75 \mathrm{~m})$, com piso de concreto, cocho para fornecimento de ração $(0,95 \mathrm{~m} \times 0,35 \mathrm{~m})$ e bebedouro com boia, dispostos dois animais do mesmo tratamento/por baia.

Foram estabelecidas duas fases: dos 90 dias até os animais atingirem a puberdade (fase 1) e a partir da puberdade até 1 ano de idade (fase 2). A idade à puberdade (dias), foi considerada quando as cordeiras aceitaram o cortejo e monta do macho vasectomizado.

Para a fase 1, foram formuladas duas dietas. Sendo, uma para obtenção de ganho de peso médio diário de aproximadamente $150 \mathrm{~g}$ (tratamento Baixo); e outra para ganho de peso médio diário próximo de $250 \mathrm{~g}$ (tratamento Alto). $\mathrm{Na}$ fase 2, uma dieta equilibrada idêntica foi fornecida para ambos os grupos.

Os ingredientes utilizados na formulação das dietas constam na Tabela 1. As dietas foram formuladas segundo o National Research Council - NRC (2007), de forma a atender as exigências dos animais durante todo o período experimental. As cordeiras foram alimentadas duas vezes ao dia (às $8 \mathrm{~h} 00$ e às 15h00), com acesso ad libitum às dietas e à água.

Os teores de matéria seca (MS), proteína bruta (PB), nutrientes digestíveis totais (NDT), extrato etéreo $(E E)$, matéria mineral $(\mathrm{MM})$, fibra em detergente neutro (FDN) e fibra em detergente ácido (FDA) das amostras do feno e da ração foram analisados pelo Laboratório de Bromatologia do Departamento de Nutrição e Melhoramento Animal da FMVZ-UNESP, Botucatu e os resultados analisados de acordo com metodologia descrita por Silva e Queiroz (2002). 
Tabela 1. Formulação e composição das dietas experimentais

\begin{tabular}{lrrr}
\hline \multirow{2}{*}{ Item } & \multicolumn{2}{c}{${\text { Fase } 1^{2}}^{2}$} & ${\text { Fase } 2^{3}}^{3}$ \\
\cline { 2 - 4 } & Baixa & Alta & Baixa/ Alta \\
\hline Feno "coastcross" & 62,45 & 20,20 & 18,81 \\
Milho moído & 23,58 & 57,23 & 68,85 \\
Farelo de Soja & 11,84 & 20,20 & 9,87 \\
Ureia & - & - & 4,80 \\
Cloreto de amônia $_{\text {Calcário calcítico }}$ & 0,00 & 0,00 & 0,05 \\
Sal mineral $^{1}$ & 0,61 & 0,10 & 0,10 \\
Monensina sódica & 1,21 & 1,04 & 1,07 \\
\hline MS & 0,30 & 0,30 & 0,30 \\
PB & 90,81 & 89,55 & 89,84 \\
NDT & 11,34 & 16,04 & 14,20 \\
EE & 65,12 & 78,20 & 79,98 \\
MM & 1,70 & 3,44 & 5,16 \\
FDN & 5,46 & 4,53 & 4,73 \\
FDA & 56,39 & 28,00 & 25,91 \\
TCA & 37,44 & 17,05 & 9,63 \\
\hline
\end{tabular}

${ }^{1}$ Composição do Sal Mineral (kg do produto) Ca 155g, P 65g, Mg 6g, S 12g, Na 115g, Se 27mg, Cu 100mg, Fe 1000mg, Zn 6000mg, Mn 1400mg, I 175mg, Co 175mg, Fl 650mg.

${ }^{2}$ Dos 90 dias de idade até o início da puberdade.

${ }^{3}$ Da puberdade até 1 ano de idade.

A cada 14 dias foram realizadas a pesagem individual dos animais com uso de balança digital, sendo o peso vivo dado em quilograma $(\mathrm{kg})$ e o ganho de peso médio diário (GPMD) em gramas (g). O GPMD foi calculado considerando o ganho das cordeiras durante 0 período, dividido pelo número de dias entre as pesagens (14 dias). Ressalta-se que foi considerado como condição experimental a similaridade do peso inicial dos animais para que fosse possível observar o efeito que cada tratamento poderia exercer ao longo do experimento.

O consumo diário de matéria seca (CMS) foi calculado para cada indivíduo e expresso em $\mathrm{kg} / \mathrm{dia}$, sendo a quantidade fornecida ajustada de acordo com as sobras diárias, que deveriam ser de $10 \%$ da quantidade oferecida no dia anterior. No mesmo dia da pesagem, foi determinado o escore de condição corporal (ECC) por meio de palpação da região lombar, com atribuição de notas fracionadas em 0,25 , sendo, 1 para animais excessivamente magros e 5 para aqueles extremamente gordos (SAÑUDO; SIERRA, 1986).

Foram realizadas colheitas de sangue da veia jugular a cada 28 dias durante todo o período experimental. Para colheita utilizou-se tubos heparinizados. Posteriormente, o sangue foi centrifugado a $700 \mathrm{G}$ por 15 minutos para obtenção do plasma. As amostras de plasma foram armazenadas em alíquotas de $1 \mathrm{~mL}$ e congeladas a $-20^{\circ} \mathrm{C}$ até serem analisadas.

A determinação das concentrações plasmáticas de insulina $(\mu \mathrm{IU})$ e $\mathrm{P}_{4}(\mathrm{ng} / \mathrm{mL})$ foram realizadas no Laboratório de Endocrinologia da FMVZ- UNESP, Botucatu por meio da técnica de Radioimunoensaio (RIA), utilizando-se kits comerciais (Progesterone Beckman Coulter ${ }^{\circ}$ e Insulin IRMA Kit Immunotech Beckman Coulter, República Tcheca) e seguindo recomendações dos fabricantes. Todas as amostras foram analisadas em duplicata.

0 experimento foi conduzido em delineamento inteiramente casualizado, composto por oito repetições e dois tratamentos, sendo considerado diferenças estatisticamente significativas quando $P<0,05$. $O$ modelo estatístico utilizado foi o modelo de efeitos misto devido a observação dos efeitos fixos (tratamento, tempo e a interação entre eles) e aleatórios do animal (SEARLE et al., 1992). As análises estatísticas foram realizadas pelo software estatístico SAS versão 9.3 (SAS, 2003) e o modelo de efeitos mistos foi ajustado utilizando o procedimento PROC MIXED. Para encontrar quais os tempos que diferem estatisticamente entre si, utilizou-se o teste ajustado de TukeyKramer para comparações múltiplas de médias em modelos mistos. 


\section{RESULTADOS}

Foram observadas diferenças entre os tratamentos para as variáveis peso vivo e escore de condição corporal $(P>0,05)$, sendo as cordeiras submetidas à dieta de alta velocidade de crescimento as tiveram desenvolvimento superior durante toda a fase experimental, entretanto, não houve diferença para o ganho de peso médio diário e consumo diário de matéria seca, conforme demonstra a Tabela 2 .

Tabela 2. Médias e desvio padrão dos pesos e escore de condição corporal iniciais e finais e consumo diário de matéria seca das cordeiras Bergamácia submetidas aos tratamentos Baixo e Alto nas duas fases experimentais

\begin{tabular}{|c|c|c|c|c|}
\hline \multirow{2}{*}{ Variáveis } & \multicolumn{2}{|c|}{ Fase $1^{1}$} & \multicolumn{2}{|c|}{ Fase $2^{2}$} \\
\hline & Baixo & Alto & Baixo & Alto \\
\hline Peso Inicial (kg) & $14,95 \pm 1,98$ & $13,90 \pm 1,53$ & $29,39 \pm 3,09$ & $38,55 \pm 2,86^{*}$ \\
\hline Peso Final (kg) & $28,77 \pm 3,62$ & $36,95 \pm 3,00 *$ & $57,17 \pm 4,09$ & $65,17 \pm 3,86^{*}$ \\
\hline $\begin{array}{l}\text { Ganho de peso médio } \\
\text { diário }(\mathrm{g})\end{array}$ & $140,00 \pm 0,06$ & $235,00 \pm 0,09 *$ & $174,00 \pm 0,07$ & $170,00 \pm 0,05$ \\
\hline $\begin{array}{l}\text { Consumo Diário de Matéria } \\
\text { Seca }(\mathrm{g})\end{array}$ & $0,83 \pm 0,14$ & $0,96 \pm 0,07$ & $1,23 \pm 0,08 *$ & $1,08 \pm 0,08$ \\
\hline Escore de Condição Corporal (1-5) & $1,42 \pm 0,51$ & $1,74 \pm 0,76$ & $2,76 \pm 0,24$ & $3,12 \pm 0,22 *$ \\
\hline
\end{tabular}

${ }^{1}$ Dos 90 dias de idade até ao início da puberdade.

${ }^{2}$ Da Puberdade até 1 ano de idade.

$* P<0,05$

Houve efeito do mês e do tratamento nos níveis plasmáticos de insulina $(\mathrm{P}<0,05)$. Contudo, a interação entre mês e tratamento foi nãosignificativa $(P>0,05)$. Quanto as concentrações plasmáticas de $\mathrm{P}_{4}$, não houve efeito do tratamento e da interação entre mês e tratamento $(P>0,05)$, sendo observado influência apenas do mês $(P<0,05)$.

Tabela 3. Médias e desvio padrão das concentrações plasmáticas da insulina ( $\mu \mathrm{IU})$ e progesterona $(\mathrm{ng} / \mathrm{mL})$ das cordeiras Bergamácia submetidas aos tratamentos Baixo e Alto durante o experimento

\begin{tabular}{lcccc}
\hline \multirow{2}{*}{ Momento } & \multicolumn{2}{c}{ Insulina } & \multicolumn{2}{c}{ Progesterona } \\
\cline { 2 - 5 } & Baixo & Alto & Baixo & Alto \\
\hline 1 & $1,03 \pm 0,23$ & $1,20 \pm 0,24$ & $0,15 \pm 0,05$ & $0,05 \pm 0,05$ \\
2 & $0,83 \pm 0,29$ & $0,93 \pm 0,40$ & $0,23 \pm 0,11$ & $0,07 \pm 0,06$ \\
3 & $0,58 \pm 0,28$ & $0,53 \pm 0,37$ & $0,15 \pm 0,09$ & $0,07 \pm 0,05$ \\
4 & $0,66 \pm 0,26$ & $0,95 \pm 0,21^{*}$ & $0,11 \pm 0,06$ & $0,29 \pm 0,10$ \\
5 & $0,69 \pm 0,29$ & $0,88 \pm 0,22$ & $0,42 \pm 0,07$ & $1,21 \pm 1,00$ \\
6 & $0,72 \pm 0,22$ & $1,04 \pm 0,21^{*}$ & $0,70 \pm 0,20$ & $1,26 \pm 0,48$ \\
7 & $1,06 \pm 0,37$ & $1,17 \pm 0,06$ & $0,92 \pm 0,09$ & $1,75 \pm 0,09$ \\
8 & $1,00 \pm 0,26$ & $1,15 \pm 0,26$ & $0,95 \pm 0,14$ & $1,48 \pm 0,27$ \\
\hline Análise de Variância & $\mathrm{F}$ & Valor $\mathrm{P}$ & $\mathrm{F}$ & Valor P \\
Efeito Tratamento & 6,97 & $<0,05$ & 3,90 & $>0,05$ \\
Efeito Mês & 8,23 & $<0,05$ & 4,58 & $<0,05$ \\
Tratamento*Mês & 0,71 & $>0,05$ & 0,70 & $>0,05$ \\
\hline
\end{tabular}

${ }^{1}$ Momento: intervalo entre cada colheita (28 dias).

${ }^{*} \mathrm{P}<0,05$

\section{DISCUSSÃO}


As cordeiras do tratamento Alto foram submetidas a elevado nível nutricional, com maior concentração energética, isto provavelmente proporcionou maior produção de propionato, que é um importante precursor para a gliconeogênese no fígado, aumentando assim a disponibilidade de nutrientes ao organismo (IGNÁCIO; CLARK, 2003), resultando em melhor ganho de peso final nas duas fases do experimento, com aumentos de $23,02 \mathrm{~kg}$ e $26,62 \mathrm{~kg}$ em relação ao peso inicial, nas fases $1 \mathrm{e}$ 2 , respetivamente.

Contudo, não houve diferença para o CMS e GPMD entre os grupos experimentais. De acordo com Mertens (1994) o CMS e consequentemente o GPMD, pode ser regulado pelo requerimento animal. Assim, a exigência energética das cordeiras do tratamento Alto pode ter sido alcançada com níveis menores de ingestão da dieta, não havendo diferença entre os tratamentos.

As cordeiras do tratamento Alto apresentaram também maior concentração plasmática de insulina, isto ocorreu provavelmente devido ao melhor status nutricional proporcionado pelo manejo nutricional imposto nas cordeiras desse tratamento, pois dietas com maior quantidade de concentrado proporciona aumento da disponibilização de propionato no rúmen como resultado da fermentação microbiana estimulando, assim, a produção de insulina (BARBOSA et al., 2011). Portanto, quanto mais energética é a dieta, maior a concentração de insulina liberada pelo pâncreas.

Assim, a concentração plasmática de insulina correspondeu ao balanço energético e padrão de homeostase dos animais avaliados. Segundo Roche et al. (2008) a insulina apresenta relação positiva com o desenvolvimento corporal, apresentando papel central na regulação do crescimento. Em estudos realizados com ovinos, foi verificado que os animais que receberam dieta rica em energia e que apresentaram maior ganho de peso e ECC foram os que também demonstraram as maiores concentrações de insulina no sangue (CALDEIRA et al., 2007).

\section{REFERÊNCIAS}

BARBOSA, F.A.; ANDRADE, V.J.; SOUZA, R.C.; GRAÇA, D.S.; PINTO, P.F.B. Dietas de alto concentrado para terminação de bovinos de corte. In: Encontro dos Médicos Veterinários e
Não houve diferença para a concentração plasmática de $\mathrm{P}_{4}$ entre os tratamentos durante todo o período experimental. Segundo Parr et al. (1993), dietas com elevada quantidade de concentrado podem afetar desfavoravelmente os níveis plasmáticos de $\mathrm{P}_{4}$ de ovelhas, pois promovem maior ingestão de matéria seca, produção e disponibilização do propionato, ganho de peso e aumento do fluxo sanguíneo hepático, causando maior clearance de $\mathrm{P}_{4}$, $\mathrm{O}$ que resulta em diminuição da concentração desse hormônio na circulação.

Portanto, esperar-se-iam diferenças nas concentrações do $\mathrm{P}_{4}$, sendo as cordeiras do tratamento Alto as que provavelmente apresentariam os menores valores durante o período o estudo, porém apesar do maior desenvolvimento corporal alcançado pelas cordeiras desse tratamento, o plano nutricional pode não ter sido tão rigoroso e, este por si só, não é foi suficiente para provocar alterações na concentração plasmática de $\mathrm{P}_{4}$. Corroborando Indu et al. (2015) que também não obtiveram diferença na concentração plasmática de $\mathrm{P}_{4} \mathrm{em}$ ovelhas da raça Malpura submetidas a dietas com diferentes proporções de volumoso:concentrado (50:50, 40:60 e 30:70).

Segundo Sasa et al. (2002) a concentração plasmática de $\mathrm{P}_{4}$ em borregas apresenta valores que variam de 0,08 a 7,36 $\mathrm{ng} / \mathrm{mL}$ ao longo do ciclo estral. Dessa forma, sugere-se que a função da secreção de $\mathrm{P}_{4}$ esteja mais relacionada à adequação da condição corporal observada nos dois grupos experimentais do que às condições nutricionais impostas durante o experimento.

\section{CONCLUSÃO}

A alimentação de cordeiras Bergamácia com um plano moderado de nutrição proporciona a produção de animais com desenvolvimento corporal e perfil hormonal satisfatórios e podendo ser considerada como estratégia de criação e otimização do manejo nutricional durante a fase de crescimento.

Zootecnistas dos Vales Do Mucuri, Jequitinhonha e Rio Doce, 32. 2011. Anais... Teófilo Otoni: [s.n.], 2011. 1 CD-ROM.

CALDEIRA, R.M.; BELO, A.T.; SANTOS, C.C.; VASQUEZ, M.I.; PORTUGUAL, A.V. The effect of 
long-term feed restriction and over-nutrition on body condition score, blood metabolites and hormonal profiles in ewes. Small Ruminant Research, v.68, p.242-255, 2007. https://doi.org/10.1016/i.smallrumres.2005.08.0 26

EVERSOLE, D.E.; BROWNE, M.F.; HALL, J.B.; DIETZ; R.E. Body condition scoring beef cows. 2000. Disponível em: http://pubs.ext.vt.edu/400/400795/400-795.html. Acesso em: 30 jun. 2016.

IGNÁCIO, R.I.; CLARK, J.H. Usefulness of ionophores for lactating dairy cows: a review. Animal Feed Science and Technology, v.106, p.39-57, 2003. https://doi.org/10.1016/S03778401(03)00065-8

INDU, S.; SEJIAN, V.; KUMAR, D.; PAREEK, A.; NAQVI; S. Ideal proportion of roughage and concentrate for Malpura ewes to adapt and reproduce in a semi-arid tropical environment. Tropical Animal Health and Production, v.47, p.1487-1495, 2015. https://doi.org/10.1007/s11250-015-0889-1

MERTENS, D.R. Regulation of forage intake. In: National Conference On Forage Quality, Evaluation, And Utilization. 1994. Anais... Lincoln:University of Nebraska, 1994. p.450-493.

NATIONAL RESEARCH COUNCIL. Nutrient requirements of dairy cattle. 7.ed. Washington, 2001. 381p.

PARR, R.A.; DAVIS, I.F.; MILES, M.A.; SQUIRES, T.J. Feed intake affects metabolic clearance rate of progesterone in sheep. Research in Veterinary Science, v.55, p.306-10, 1993. Doi: 10,1016 / 0034-5288 (93) 90099-2

PIRES, A.V.; RIBEIRO, C.V.D.M. Aspectos da nutrição relacionados à reprodução. In: BERCHIELLI, T.T.; PIRES, A.V.; OLIVEIRA, S.G.
Nutrição de ruminantes. 2. ed. Jaboticabal: FUNEP, 2011. p.513-537.

ROCHE, J.R.; BLACHE, D.; KAY, J.K.; MILLER, D.R.; SHEAHAM, A.J.; MILLER, D.W. Neuroendocrine and physiological regulation of intake with particular reference to domesticated ruminant animals. Nutrition Research Reviews, v.21, p.207-234, 2008.

\section{https://doi.org/10.1017/S0954422408138744}

SAÑUDO, C.; SIERRA, I. Calidad de la canal en la especie ovina. Ovino, v.1, p.127-153, 1986.

SAS. User's Guide. Cary, NC: SAS Institute, 2003. $129 \mathrm{p}$.

SASA, A.; TESTON, D.C.; CRIVELLENTI, T.L; RODRIGUES, P.A; DA SILVA, E.C.F; COELHO, L.A. Concentrações plasmáticas de progesterona em borregas lanadas e deslanadas durante o período de abril a novembro no estado de São Paulo. Revista Brasileira de Zootecnia, v.31, p.11501156, 2002. https://doi.org/10.1590/S1516$\underline{35982002000500011}$

SEARLE, S.R.; CASELLA, G.; MCCULLOCH, C.E. variance components. 1. ed. New York: John Wiley and Sons, 1992. 501p.

SILVA, D.J.; QUEIROZ, A.C. Análise de alimentos (Métodos Químicos e Biológicos). 2. ed. Viçosa: Universidade Federal de Viçosa. 2002. 235p.

Recebido para publicação em 16/12/2016

Revisado em 16/02/2017

Aceito em 27/03/2017 